\title{
Evaluation for hypocenter estimation error in the southwestern Kuril trench using Japan and Russia joint seismic data
}

\author{
Masayoshi Ichiyanagi ${ }^{1}$, Valentin Mikhaylov², Dmitry Kostylev ${ }^{2,3}$, Yuri Levin ${ }^{2}$ and Hiroaki Takahashi ${ }^{*}$ (i)
}

\begin{abstract}
The southwestern Kuril trench is seismically active due to the subduction of the Pacific plate. Great earthquakes in this zone have frequently induced fatal disasters. Seismic monitoring and hypocenter catalogs provide fundamental information on earthquake occurrence and disaster mitigation. Real-time hypocenter and magnitude estimates are extremely crucial data for tsunami warning systems. However, this region is located in the international border zone between Japan and Russia. The Japan Meteorological Agency and Russian Academy of Sciences have routinely determined hypocenters and issued earthquake information independently. Waveform data have not yet been exchanged internationally in real time. Here, we evaluated how a hypothetical Japan-Russia joint seismic network could potentially improve the hypocenter estimation accuracy. Experiments using numerical and observed data indicated that the joint network extended the distance over which hypocenters can be accurately determined over $100 \mathrm{~km}$ eastward compared to the Japan network only. This fact suggests that joint seismic data have the potential to improve the hypocenter accuracy in this region, which would provide improved performance in gathering disaster information at the moment of a tsunami warning.
\end{abstract}

Keywords: The southwestern Kuril trench, Hypocenter determination, Japan and Russia joint seismic network, Great subduction earthquakes, Tsunami warning

\section{Introduction}

The southwestern Kuril trench is one of the most seismically active regions in the world. The subduction of the Pacific plate at a rate of $\sim 9 \mathrm{~cm} /$ year has resulted in the frequent generation of large earthquakes. Great earthquakes of moment magnitude $(\mathrm{Mw})>8.0$, e.g., the 1963 Mw8.3 interplate and 1994 Mw8.2 intraslab earthquakes, have induced strong ground shaking and generated tsunamis, causing severe destruction (Fig. 1). The Headquarters for Earthquake Research Promotion (HERP) of the Japanese government (2020) evaluated earthquake occurrence probabilities reaching as high as $80 \%$ for

\footnotetext{
*Correspondence: hiroaki@sci.hokudai.ac.jp

${ }^{1}$ Institute of Seismology and Volcanology, Faculty of Science, Hokkaido University, Sapporo, Japan

Full list of author information is available at the end of the article
}

$M>7.8$ earthquakes over the next 30 years in this region. In addition, a possible megathrust earthquake of $M>8.8$ has been anticipated with a high (7-40\%) likelihood probability by HERP. These extremely high occurrence probabilities for large earthquakes in the future require the implementation of disaster prevention and mitigation measures to reduce the damage caused by earthquakes and tsunamis.

Tsunami inundation simulations have indicated that a tsunami produced by a subduction earthquake can arrive at the shore $20 \mathrm{~min}$ after the earthquake (Hokkaido Local Government 2012). In this event, a tsunami warning is issued primarily using the hypocenter location and magnitude. A reliable and instant hypocenter determination is required for reliable tsunami alerts.

The southwestern Kuril region is a peripheral area between Japan and Russia. Both Japanese and Russian 


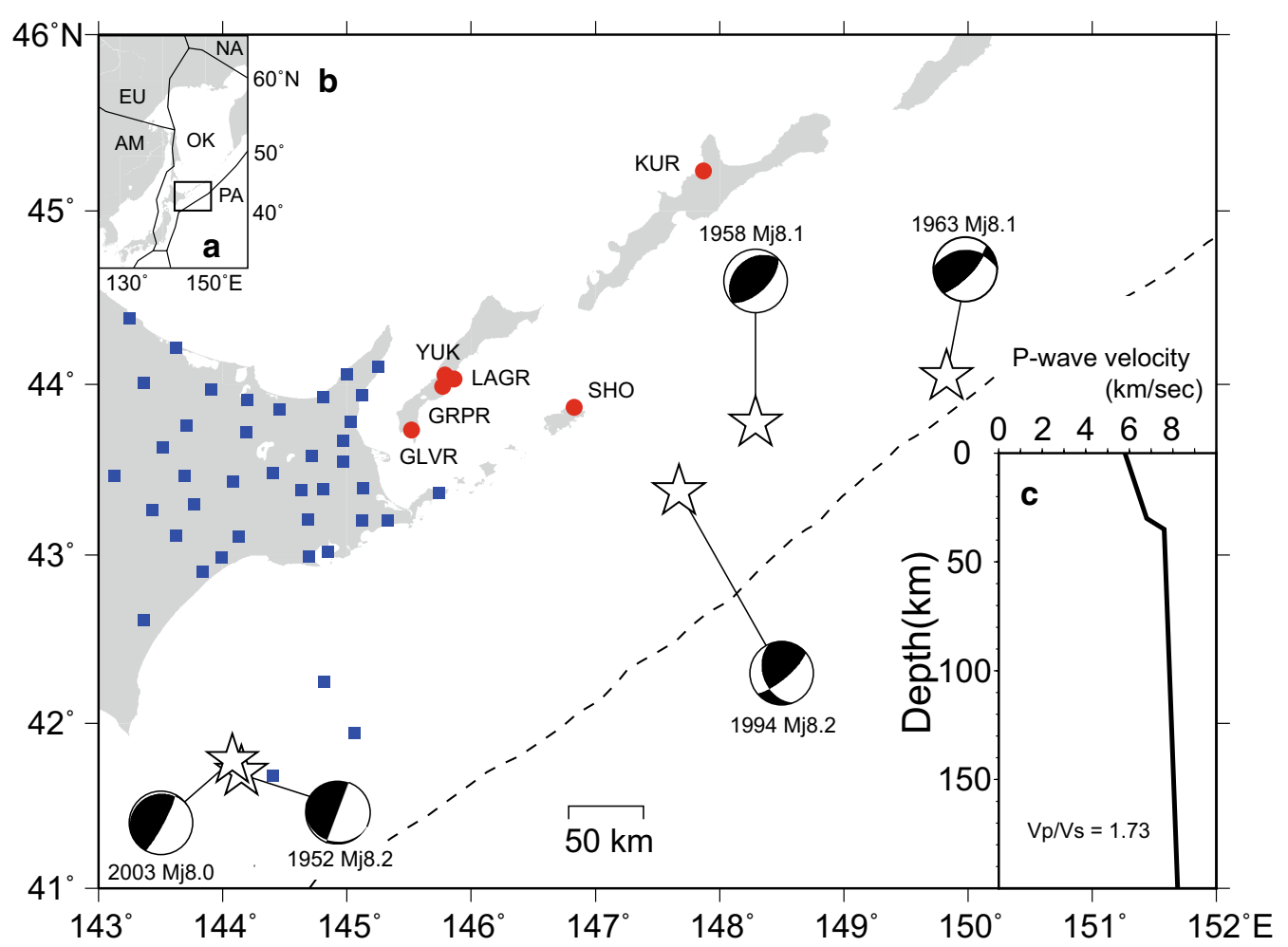

Fig. 1 Seismological stations operated by Japan and Russia. a Tectonic setting of target region. PA, Pacific plate, OK, Okhotsk plate, AM, Amur plate; EU, Eurasian plate; NA, North American plate. b Seismic station distribution; blue: Japanese station; red: Russian stations. Epicenters of great earthquakes were from Japan Meteorological Agency, and mechanism of the 1952 was from Ichikawa (1971), the 1958 was from Fukao and Furumoto (1979), the 1963 was from Kanamori (1970), and others were from Global CMT project (Global CMT Web Page, 2020). c The P-wave seismic velocity structure

institutions operate regional seismological networks in this area (Fig. 1). The Japanese dense seismic network is managed by Hokkaido University, the Japan Meteorological Agency (JMA), the National Research Institute for Earth Science and Disaster Resilience (NIED), and the Japan Agency for Marine-Earth Science and Technology (JAMSTEC) (Fig. 1). All waveform data are transmitted to the data center and exchanged in real time among Japanese institutions. The JMA performs waveform monitoring and hypocenter location services in real time to issue earthquake information. Tsunami alerts are issued by the JMA 3 min after an earthquake is detected.

The Sakhalin Branch of the Geophysical Survey (SBGS), Russian Academy of Sciences, operates the seismic network in the Russian Far East. The SBGS has deployed seismic stations on three residential islands along the southwestern Kuril trench (Fig. 1). Real-time waveform monitoring and hypocenter estimation are conducted routinely. Russian authorities issue tsunami warnings based on hypocenter information from the SBGS.

The distribution of islands determines the geographical configuration of seismic networks (Fig. 1). The extents of national territory might also control the geometry of seismic networks. Consequently, Japanese and Russian stations occupy the western and eastern parts of this region, respectively (Fig. 1). A hypothetical joint seismic network would improve the network configuration greatly. Accordingly, Hokkaido University and the SBGS have been in cooperation to exchange seismic data. Here, we evaluate the hypocenter determination performance using the Japan-Russia international joint seismic network with both synthetic and observed seismic data.

\section{Hypocenter determination experiment by synthetic travel time data Synthetic travel time data}

A hypothetical Japan-Russia joint seismic network might have the potential to improve hypocenter errors. Numerical experiments of hypocenter determination using synthetic travel time data were performed. The experimental procedures were as follows: (1) the theoretical travel times of $\mathrm{P}$ - and $\mathrm{S}$-waves were calculated at each seismic station from a given virtual hypocenter; (2) statistical random errors were added to the travel times from 
step (1); and (3) the hypocenter locations were estimated using the travel time data from step (2).

Virtual hypocenters were distributed at grid points diagonally along the trench between E145 and E151 degrees (Figs. 1 and 2). Hypocenter depths offshore and near islands were set to $10 \mathrm{~km}$ and $100 \mathrm{~km}$, respectively. A total of 12 virtual hypocenters were given. Theoretical travel times at each station were calculated for each 12 hypocenters using Hirata and Matsu'ura's (1987) algorithm. The one-dimensional seismic velocity structure model shown in Fig. 1 is applied.

Although observed travel times involve random and systematic errors, pseudo-errors were added to the theoretical travel times. Phase reading errors were simulated using a Gaussian probabilistic function with a variance of $1 \mathrm{~s}$ for P-wave arrival times and $2 \mathrm{~s}$ for S-wave arrival times. Velocity structure errors were given as a function of the hypocentral distance: a Gaussian probabilistic function with a variance of $0 \mathrm{~s}$ for epicentral distances less than $25 \mathrm{~km}, 0.05 \mathrm{~s}$ for $25-50 \mathrm{~km}, 0.10 \mathrm{~s}$ for $50-100 \mathrm{~km}, 0.20 \mathrm{~s}$ for $100-200 \mathrm{~km}$, and $0.10 \mathrm{~s}$ for every $100 \mathrm{~km}$ increment thereafter. Each virtual hypocenter produced a total of 90 travel time data points with statistical random errors. In addition to the direct S-wave, arrival phases of converted and refracted waves may include in actual waveform data. It is necessary to carefully read the $\mathrm{S}$-wave arrivals taking into account the travel time residual.
Hypocenters were determined using simulated travel time data processed using Hirata and Matsu'ura's (1987) nonlinear inversion program. This algorithm uses a given hypocenter location and its errors as prior information for maximum likelihood estimation. Iterations were calculated using the previous hypocenter location and station corrections, and 10 iterations were performed.

\section{Reproducibility of shallow virtual earthquakes}

Comparisons were evaluated between the given and estimated hypocenters using the Japanese network only and the Japan-Russia joint network. First, we tested the reproducibility of the given virtual hypocenters with a depth of $10 \mathrm{~km}$ in the offshore region in Fig. 2 and Additional file 1: Fig. S1. Figure 2a indicates the estimated hypocenters using only the Japanese seismic network. One given hypocenter produced 90 estimated hypocenters.

The Japanese network was capable of determining the hypocenters to the west of latitude E146 (Fig. 2a). Although the horizontal hypocenter locations west of E147 were well estimated, the depth varied widely. The variance in both the depth and the horizontal location to the east of latitude E148 suggested low reliability.

The Japan-Russia joint network data successfully improved the hypocenter error. The hypocenter distribution shown in Fig. $2 \mathrm{~b}$ using the joint network clearly indicates an obvious reduction in the variance at E147, as

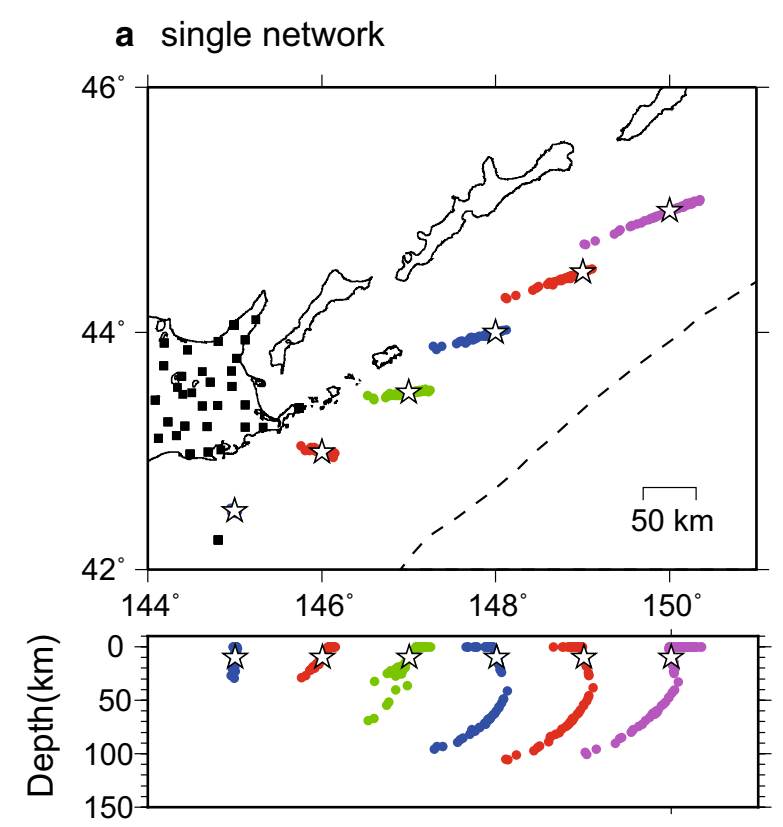

\section{b joint network}

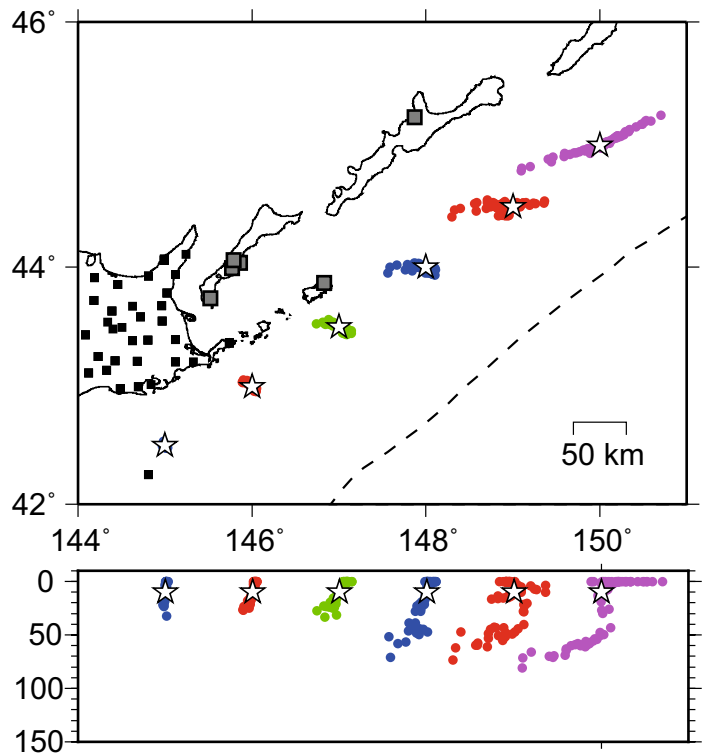

Fig. 2 Simulated hypocenter determination using synthetic travel time data. The given hypocenters at each grid are shown by open stars. a Calculated hypocenters for given shallow (10 km depth) earthquakes using the Japan network only. b Same as a but using the Japan-Russia joint network data. The 90 travel times and hypocenters were produced from one given virtual hypocenter 
shown in Fig. 2a. The hypocenter variance at E148 was also improved. These conclusions can also be found in the standard deviation data shown in Table 1. Remarkable error reductions were identified on all components on the grids at E147 and E148. Table 1 also suggests good estimation results on the latitudinal component but less accurate estimates on the longitudinal component. The depth accuracy for the hypocenters to the east of E148 was still poor and had no resolution. The horizontal hypocenter locations at E149 and E150 were not improved and were unreliable. These results suggest that the joint network is able to give reliable horizontal locations to the west of E148. The depth component might be valid to the west of E147.

\section{Reproducibility of intermediate-depth virtual earthquakes}

The same test was performed for virtual earthquakes at an intermediate depth $(100 \mathrm{~km})$ in the nearshore region (Fig. 3 and Additional file 1: Fig. S2). The Japanese network can determine the depth component well to the west of E147 (Fig. 3a, Table 1). The horizontal components, however, showed large variance at E147. The deviation from the true hypocenter increased both horizontally and vertically to the east of E148.

The Japan-Russia joint network provided remarkable reductions in the variance in the latitudinal and depth components on the grids at E147 and E148 (Fig. 3b, Table 1). The depth component was also improved at E149 and E150. However, the longitudinal component did not improve much at E148 and to the east. The errors on the longitudinal component for these intermediatedepth earthquakes were approximately twice as large as those for the shallow-depth earthquakes.

\section{Summary of numerical experiments}

The numerical experiments using synthetic travel time data suggested the following features: (1) the joint network can reduce hypocenter errors, (2) the joint network can determine reliable hypocenters to the west of E147 for all earthquakes, (3) shallow- and intermediate-depth earthquakes are simulated with relatively large errors on the depth and longitudinal components, respectively, and (4) even the joint network cannot give an adequate accuracy to distinguish hypocenter depths to the east of E148. These facts indicate that compared with the Japan network alone, the joint network has the capability to determine reliable hypocenters more than $100 \mathrm{~km}$ to the east.

Hypocenter determination using observed travel time data Seismic network and observed data

The determination of hypocenters was tests using observed seismological data. The Japanese seismic network is distributed on Hokkaido with a high density (station spacing of approximately $20 \mathrm{~km}$ ) (Fig. 1). Data from three cabled ocean bottom seismographs operated by JAMSTEC are also included. Only stations in eastern Hokkaido were applied to reduce the travel time error due to the anomalous seismic velocity structure in central Hokkaido (e.g., Kita et al. 2012).

The Russian stations are deployed on three residential islands: three stations on Kunashir Island and one station each on the islands of Itrup and Sikotan (Fig. 1). No stations are operated to the east of station KUR because the islands are uninhabited. The maximum interstation distance between stations SHO and KUR is approximately $200 \mathrm{~km}$.

Digital continuous waveform data with GPS time stamps are synchronously recorded at both the Japanese and the Russian seismic networks. The JMA

Table 1 Standard deviations of the estimated hypocenter locations at each grid point

\begin{tabular}{|c|c|c|c|c|c|c|c|c|}
\hline \multirow[t]{7}{*}{10 km depth } & Grid point (Deg.) & $145.0 / 42.5$ & $146.0 / 43.0$ & $147.0 / 43.5$ & $148.0 / 44.0$ & $149.0 / 44.5$ & $150.0 / 45.0$ & $151.0 / 45.5$ \\
\hline & SD (Lon./Lat./Dep.) & 1 & 8 & 15 & 17 & 21 & 29 & 38 \\
\hline & Single network & 1 & 0 & 1 & 2 & 4 & 6 & 8 \\
\hline & $(\mathrm{km})$ & 10 & 9 & 17 & 45 & 48 & 40 & 40 \\
\hline & SD (Lon./Lat./Dep.) & 1 & 4 & 8 & 11 & 20 & 32 & 34 \\
\hline & Joint network & 1 & 2 & 2 & 2 & 3 & 6 & 8 \\
\hline & $(\mathrm{km})$ & 9 & 11 & 12 & 24 & 34 & 33 & 37 \\
\hline \multirow[t]{7}{*}{100 km depth } & Grid point (Deg.) & $145 / 43.5$ & $146.0 / 44.0$ & $147.0 / 44.5$ & $148.0 / 45.0$ & $149 / 45.5$ & $150 / 46.0$ & $151 / 46.5$ \\
\hline & SD (Lon./Lat./Dep.) & 5 & 19 & 32 & 37 & 47 & 51 & 56 \\
\hline & Single network & 1 & 5 & 9 & 11 & 14 & 14 & 15 \\
\hline & $(\mathrm{km})$ & 1 & 3 & 17 & 41 & 32 & 37 & 38 \\
\hline & SD (Lon./Lat./Dep.) & 2 & 6 & 15 & 32 & 45 & 50 & 55 \\
\hline & Joint network & 0 & 2 & 3 & 6 & 11 & 13 & 15 \\
\hline & $(\mathrm{km})$ & 2 & 1 & 5 & 10 & 12 & 24 & 29 \\
\hline
\end{tabular}


a single network

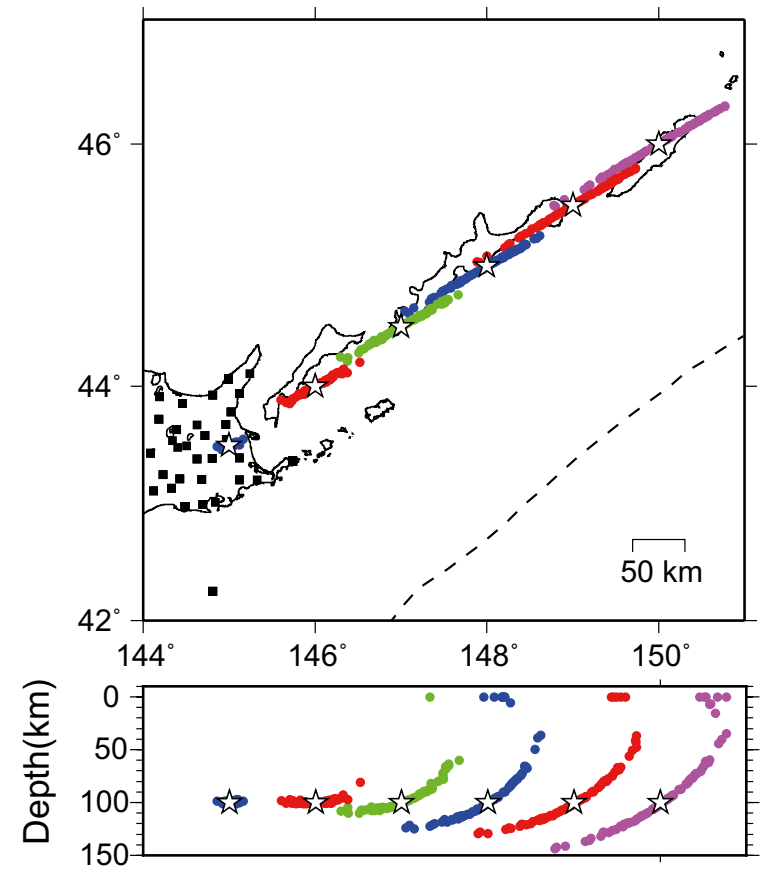

b joint network

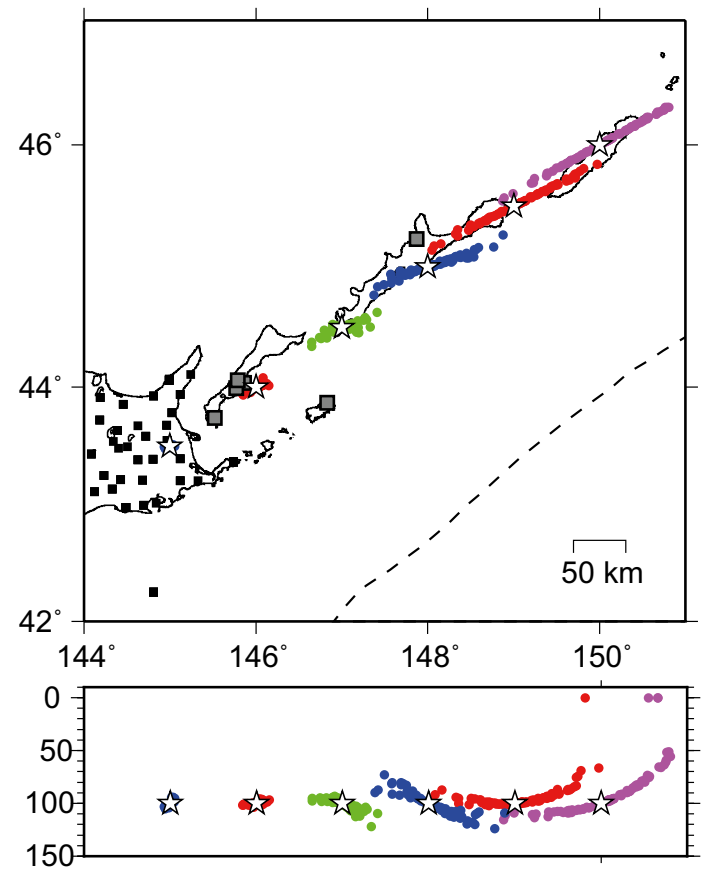

Fig. 3 Same as Fig. 2 but for intermediate (100 km depth) virtual hypocenters a using the Japan network only and $\mathbf{b}$ using the Japan-Russia joint network data

routinely reads the $\mathrm{P}$ - and S-wave arrival times from integrated waveform data from all Japanese institutions and published bulletins. The SBGS also routinely extracts the P-and S-wave arrival times for the Russian stations. Hokkaido University receives the above arrival time data from the JMA and SBGS and produces a combined arrival time catalog. The JMA and SBGS catalog registered earthquakes with magnitude more than 4.5 in common. These unified $\mathrm{P}$ - and $\mathrm{S}$-wave arrival time data were used for the hypocenter determination.

\section{Hypocenter determination from the Japan-Russia joint network observation data}

The numerical experiments suggested an improvement in the hypocenter accuracy using the joint Japan and Russia network data. Hypocenters were calculated using the observed travel time data. Earthquakes from 2010 September 7 to 2014 January 9 were applied.

The initial hypocenters were estimated using Hirata and Matsu'ura's (1987) algorithm. The 1-D seismic velocity structure was the same as that in the numerical experiments (shown in Fig. 1). A station correction was calculated for each station using the average residual between the theoretical and observed arrival times of all earthquakes. The final hypocenters were determined by an iterative procedure with the above station corrections.
Figure 4a shows a comparison of the hypocenter locations from the joint network and Japan network only. The horizontal and depth migration tendencies agree with the numerical simulation results. A comparison with USGS PDE hypocenters estimated from the global network is also shown in Fig. 4b. Hypocenters projected to perpendicular to the trench axis are also shown in Additional file 1: Fig. S3. These data suggest that the true hypocenters are shallower than those determined using the Japan network only.

\section{Discussion and conclusion}

The numerical experiments indicate that the joint network data can effectively determine reliable hypocenters to the west of E147. The variance reductions at each grid point shown in Table 1 suggest significant variance improvements, even at E148 and E149. The Russian stations strongly contribute to this upgrade. Although a hypocenter comparison between the joint network and USGS PDE data for Mw $>5.0$ earthquakes shows locations that are systematically shifted northeast from those determined by the joint network, the differences are not significant (Fig. 4b).

The data in Table 1 indicate that an earthquake with a $10 \mathrm{~km}$ depth at E148 may produce a $\pm 47 \mathrm{~km}$ depth error at the $95 \%$ confidence level $(1.96 \times \mathrm{SD})$. This indicates 


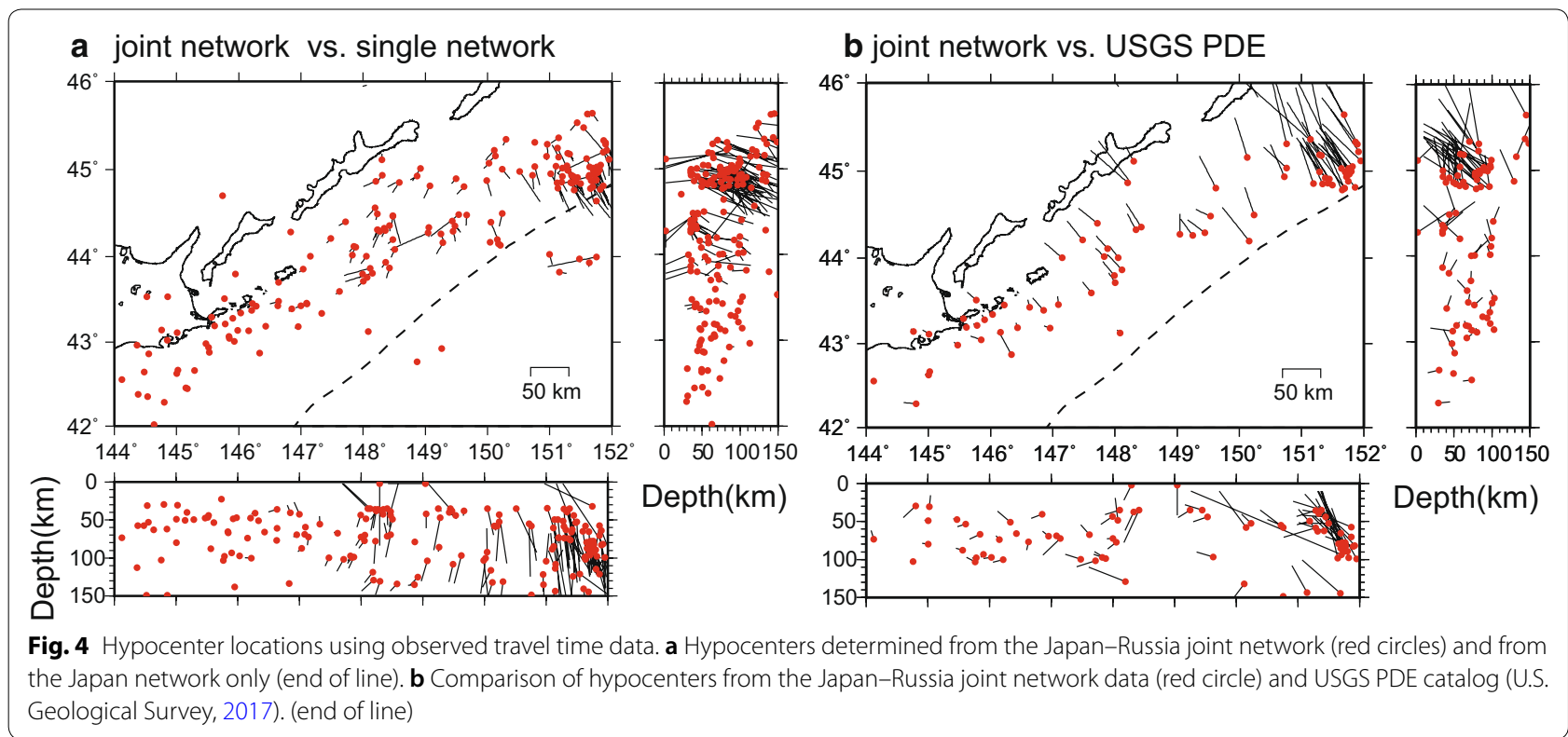

that it will be difficult to distinguish between shallow- and intermediate-depth earthquakes to the east of E148. Even the joint network reveals a trade-off between the depth component and the horizontal location in the region farther to the east. Tsunami warnings should be issued regardless of how deep the depth is calculated for the hypocenter.

The hypothetical joint seismic network gives potential errors of approximately $\pm 20 \mathrm{~km}$ on the horizontal components and $\pm 50 \mathrm{~km}$ on the depth component at the $95 \%$ confidence level for two historical great earthquakes: the JMA magnitude (Mj) 8.21994 earthquake (N43.375, E147.673, depth of $28 \mathrm{~km}$ ) and the $1958 \mathrm{Mj} 8.1$ earthquake (N43.776, E148.290, depth of $13 \mathrm{~km}$, JMA catalog). These location error ranges are sufficiently acceptable for tsunami warning systems. However, the potential errors of events farther to the east, namely, the $1963 \mathrm{Mj} 8.1$ earthquake (N44.048, E149.825, depth of $0 \mathrm{~km}$, JMA catalog), are approximately $\pm 60 \mathrm{~km}$ on the horizontal components and $\pm 70 \mathrm{~km}$ on the depth component. A maximum horizontal error of $120 \mathrm{~km}$ may cause a tsunami to arrive at the coastline more than 10 min earlier than predicted for a wave speed of $500 \mathrm{~km} / \mathrm{s}$.

Scientists usually use aftershock distributions to characterize earthquakes. The hypocenter location variance data in Table 1, however, indicate that the hypocenter locations to the east of E147 likely involve systematic errors that are too large to apply these locations for scientific purposes. For instance, the aftershock data of great earthquakes in this region will not provide clues to distinguish the true nodal planes of focal mechanisms. Heterogeneous three-dimensional velocity structure due to subduction should also be taken into account for more precise hypocenter estimation.

The joint seismological network has the potential to improve the hypocenter accuracy in the southwestern Kuril trench. Quick and reliable tsunami warnings due to anticipated great and megathrust earthquakes are required because of the high occurrence probability of such events (HERP 2017). The Japanese and Russian governmental agencies responsible for issuing tsunami warnings do not operate a regional real-time seismic data exchange at present. Experimental real-time data have been successfully transmitted between Hokkaido University (Hokkaido, Japan) and the SBGS (Sakhalin, Russia) since 2002 (Ichiyanagi et al. 2002). This feasibility study shows that the real-time exchange of seismic waveform data is technically possible.

\section{Supplementary information}

Supplementary information accompanies this paper at https://doi. org/10.1186/s40623-020-01215-0.

Additional file 1: Fig. S1. Simulated hypocenter determination using synthetic travel time data same as shown in Fig. 2 but with error bars. Note that only depth component error was indicated in depth section. Fig. S2. Same as Fig. S1 but for intermediate (100 km depth) virtual hypocenters. Fig. S3. Hypocenter locations using observed travel time data same as shown in Fig. 4 but projected to parallel and perpendicular to trench axis direction.

\section{Abbreviations}

HERP: The headquarters for earthquake research promotion of Japanese government; JAMSTEC: Japan Agency for Marine-Earth Science and Technology; JMA: Japan Meteorological Agency; Mj: Japan Meteorological Agency's magnitude; Mw: Moment magnitude; NIED: National Research Institute for Earth Science and Disaster Resilience; PDE: The Preliminary Determination of 
Epicenters; SBGS: Sakhalin Branch of Geophysical Survey of Russian Academy of Science; USGS: United States Geological Survey.

\section{Acknowledgements}

We thank two anonymous reviewers and the editor for providing comments that improved the manuscript. Japanese unified hypocenter catalog is from the Japan Meteorological Agency in collaboration with the Ministry of Education, Culture, Sports, Science and Technology using seismic wave data provided by cooperating organizations, including Hokkaido Univ., Hirosaki Univ., Tohoku Univ., the Univ. of Tokyo, Nagoya Univ., Kyoto Univ., Kochi Univ., Kyushu Univ., Kagoshima Univ., the Nat. Res. Inst. Earth Sci. Disa. Prev., the Nat. Inst. Adv. Ind. Sci. Tech., the Tokyo Metropolitan Govern., Shizuoka Pref., the Hot Springs Res. Inst. Kanagawa Pref., Yokohama City, the GSI and the JAMSTEC. Russian catalog is from Geophysical Survey of Russian Academy of Sciences. Figures were partially drawn using GMT (Wessel et al. 2013). This research was supported by JSPS KAKENHI (25257204) and the Ministry of Education, Culture, Sports, Science and Technology (MEXT) of Japan, under its The Second Earthquake and Volcano Hazards Observation and Research Program. This study was also supported by ERI JURP 2016-F2-02, 2017-F2-02, 2018-F2-02, 2019-F2-02, and 2020F2-02.

\section{Authors' contributions}

Ml created unified catalog, calculated hypocenters and MV, DK, YL created seismic wave arrival time data at SBGS. HT conceived this research, evaluated the results and wrote manuscript. All authors read and approved the final manuscript.

\section{Funding}

This research was supported by JSPS KAKENHI (25257204) and the Ministry of Education, Culture, Sports, Science and Technology (MEXT) of Japan, under its The Second Earthquake and Volcano Hazards Observation and Research Program.

\section{Availability of data and materials}

The datasets used during the current study are available from the corresponding author on reasonable request.

\section{Consent for publication}

Not applicable.

\section{Competing interests}

The authors declare that they have no competing interests.

\section{Author details}

${ }^{1}$ Institute of Seismology and Volcanology, Faculty of Science, Hokkaido University, Sapporo, Japan. ${ }^{2}$ Sakhalin Branch of Geophysical Survey, Russian
Academy of Sciences, Yuzhno-Sakhalinsk, Russia. ${ }^{3}$ Institute of Marine Geology and Geophysics, Far Eastern Branch of Russian Academy of Sciences, Yuzhno-Sakhalinsk, Russia.

Received: 8 April 2020 Accepted: 8 June 2020

Published online: 16 June 2020

\section{References}

Fukao Y, Furumoto M (1979) Stress drops, wave spectra and recurrence intervals of great earthquake of 1958 November 6. Geophys J R astr Soc. 57:23-40

Hirata N, Matsuura M (1987) Maximum-likelihod estimation of hypocenter with origin time eliminated using nonlinear inversion technique. Phys Earth Planet Int. 47:50-61

Hokkaido Local Government (2012) Tsunami inundation forecast map for the Pacific coast of Hokkaido, http://www.pref.hokkaido.lg.jp/sm/ktk/bsb/ tunami/index.htm. Accessed 2 April 2017

Ichikawa M (1971) Reanalyses of mechanism of earthquakes which occurred in and near Japan, and statistical studies on the nodal plane solutions obtained, 1926-1968. Geophys Mag 35:207-274

Ichiyanagi M., H. Takahashi, M. Kasahara, R. Sen, C. Kim (2002) The experiment of the real time waveform data exchange by internet between Sakhalin and Hokkaido, Programme and abstracts of the seismological society of Japan fall meeting, P228

Kanamori H (1970) Synthesis of long-period surface waves and its application to earthquake source studies - Kurile Islands earthquake of October 13, 1963. J Geophys Res 75:5011-5027

Kita S, Hasegawa A, Nakajima J, Okada T, Matsuzawa T, Katsumata K (2012) High-resolution seismic velocity structure beneath the Hokkaido corner, northern Japan: Arc-arc collision and origins of the 1970 M 67 Hidaka and 1982 M 71 Urakawa-oki earthquakes. J Geophys Res. https://doi. org/10.1029/2012jb009356

Global CMTWeb Page, https://www.globalcmt.org/. Accessed 7 April 2020

The headquarters for earthquake research promotion, long term probability of earthquake occurrence, http://www.jishin.go.jp. Accessed 2 April 2020

U.S. Geological Survey, the preliminary determination of epicenters (PDE) bulletin, https://earthquake.usgs.gov/data/pde.php. Accessed 2 April 2017

Wessel P, Smith WHF, Scharroo R, Luis JF, Wobbe F (2013) Generic mapping tools: improved version released, EOS Trans. AGU 94:409-410

\section{Publisher's Note}

Springer Nature remains neutral with regard to jurisdictional claims in published maps and institutional affiliations.

\section{Submit your manuscript to a SpringerOpen ${ }^{\circ}$ journal and benefit from:}

- Convenient online submission

- Rigorous peer review

- Open access: articles freely available online

- High visibility within the field

- Retaining the copyright to your article

Submit your next manuscript at $\boldsymbol{\nabla}$ springeropen.com 